\title{
Spontaneous Kidney Bleed after Acute Coronary Syndrome Treatment
}

\author{
Rima Chaddad ${ }^{1}$, Marwan Zeidan $^{2}$, Ali Chaaban ${ }^{3}$ and Souzan Tatari ${ }^{3 *}$ \\ ${ }^{1}$ Lebanese University, Lebanon \\ ${ }^{2}$ Beirut Arab University, Lebanon \\ ${ }^{3}$ Rafik Hariri University Hospital, Lebanon
}

Received: August 14, 2017; Published: September 06, 2017

*Corresponding author : Souzan Tatari, MD, Rafik Hariri University Hospital, Jnah, Beirut-Lebanon, Tel: +961 3 833847;

Email: tatary_suzan@hotmail.com

\begin{abstract}
Spontaneous kidney bleed is a rare condition. Renal tumors are the most common cause of bleeding whereas anticoagulant and antiplatelet therapy are some of the less common causes. We present a case of a 72 year old male patient with acute coronary syndrome (ACS), who developed massive painless hematuria after ACS treatment. He was found to have spontaneous right pelvicaliceal bleed by computed tomography (CT). The patient was successfully treated conservatively.
\end{abstract}

Keywords: Spontaneous Kidney Bleed; Acute Coronary Syndrome; Antithrombotic Agents

\section{Introduction}

Spontaneous kidney bleed is a diagnostic dilemma. It is a rare condition in clinical practice. Although lots of research has been done in this subject, still it remains elusive. The most common cause of bleeding are benign and malignant renal tumors, especially angiomyolipomas and renal cell carcinoma [1-3]. Other known causes are long term hemodialysis, arteriosclerosis or arthritis, renal infections and vascular lesions. Renal cysts, blood dyscrasias or anticoagulant and antiplatelet therapy are some of the less common causes [4]. Antithrombotic therapy plays a cornerstone role in the therapy of ACS. One of the most feared and serious complication of enoxaparin is retroperitoneal hemorrhage which accounts for about $5 \%$ of enoxaparin induced complications [5]. Age over 65 years, renal insufficiency, and concomitant use of aspirin are some known risk factors for retroperitoneal hemorrhage with enoxaparin [6,7].

Only a few cases with enoxaparin-induced spontaneous hemorrhage have been reported in the literature [8]. Spontaneous retroperitoneal hemorrhage could present as a rare lifethreatening emergency with sudden onset of massive bleeding [9]. Flank and back pain, hematuria, hypotension and shock are the clinical features. CT is the most valuable examination for patients with spontaneous kidney bleed [10]. The main stay treatment of spontaneous retroperitoneal hemorrhage consists of regimen modification or correction of the anticoagulation state, volume resuscitation and transfusion therapy [11]. Amano et al. [12] reported that nephrectomy should be performed immediately in patient with uncontrollable hypotension due to intractable bleeding [12]. But if an initial conservative therapy could control retroperitoneal hemorrhage, a watch-and-wait strategy may be appropriate for the patient.

\section{Case Presentation}

We report a 72 year old gentleman with a past medical history significant for long standing hypertension who presented to the emergency department for 2 episodes of oppressive chest pain lasting each one for $15 \mathrm{~min}$ that radiates to the shoulders. Electrocardiography was done showing no ST-T changes. Acute coronary syndrome was highly suspected. ACS treatment with aspirin, clopidogrel and enoxaparin was given. Coronary angiography was done via right radial approach and showed minimal atheroma. So antiplatelets and anticoagulation were stopped. Then patient developed hematuria that became massive lately at night. On the following day he had a drop in hematocrit from 33.7 to 28.5 and BP dropped from 130/77 to 76/42 mmHg. Intravenous hydration and transfusion of packed red blood cells (PRBCs) were given. An urologist was consulted and urgent CT scan of the abdomen and pelvis was performed using Philips MX 16-detecter CT scanner with $3 \mathrm{~mm}$ axial reconstructed slice thickness before and after IV contrast administration with excretory phase. Unenhanced phase showed high density in the right renal collecting system reaching the urinary bladder denoting spontaneous kidney bleeding. 
Moderate right hydronephrosis and hydroureter were observed. There were no space occupying lesions in the right kidney or calculi formation. The left kidney was unremarkable. The urinary bladder was over distended with a trabecular wall. The prostate gland was enlarged in size. Excretory phase (after 15 minutes) showed delayed contrast material excretion in the right kidney; however normal excretion was seen on the left side. Cystoscopy showed blood clots in the bladder. Ureteroscopy was done to the right side reaching the kidney calyces where active bleed was seen. A right double J and a 3-way Foley catheter were inserted. Biopsy was not done at that time due to massive bleed. Then the patient became hemodynamically stable after transfusion of a total of 3 units of PRBCs. Our patient was treated conservatively and was discharged home asymptomatically. He came later after 1 week for transurethral resection of the prostate (TURP) and transurethral resection of bladder tumor (TURBT). Biopsy of the bladder showed normal mucosa. Biopsy of the prostate showed benign prostatic hypertrophy. Except anticoagulation, no particular cause for the condition could be found. Patient was asymptomatic at 3 months follow up.

\section{Discussion}

Enoxaparin is used commonly for treatment of ACS worldwide. Enoxaparin had a low incidence of bleeding and fatal complications are rare due to retroperitoneal bleed. Only few cases were reported in the literature. Our case had shown that age and hypertension are risk factors for this patient. Even though tumors are the most common cause of spontaneous kidney bleed our CT images showed kidney free lesions with high density fluid in the right renal calyces and pelvis reaching the ureter compatible with spontaneous renal hemorrhage, moderate hydronephrosis and hydroureter (Figure 1(A-B)), (Figure 2(A-B)), (Figure 3(A-B)). Double J and a 3- way Foley catheter with irrigation were inserted. Although the patient was unstable at one point, our conservative measures which included stopping antiplatelets and anticoagulation, intravenous hydration and transfusion of PRBCs succeeded in stabilization the condition.

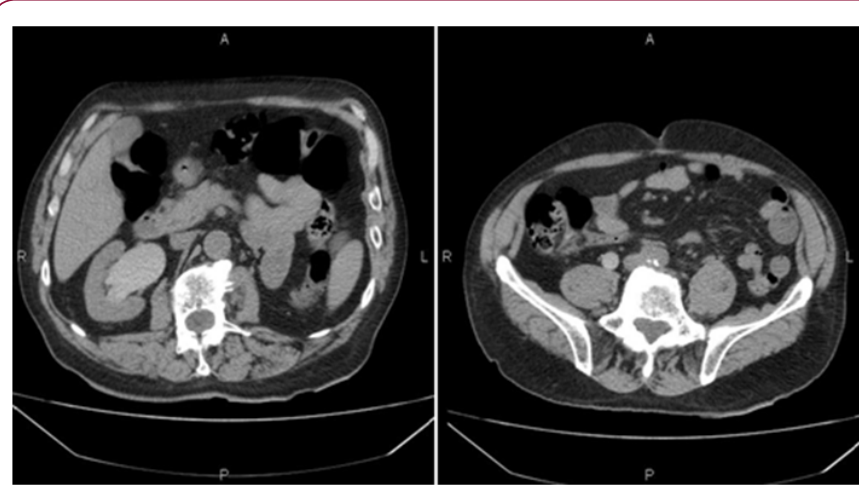

Figure 1(A-B): Unenhanced MDCT scan abdominal axial images showing high density fluid in the right renal calyces and pelvis reaching the ureter compatible with spontaneous renal hemorrhage. Moderate hydronephrosis and hydroureter were seen on the right side. No renal space occupying lesions were observed.

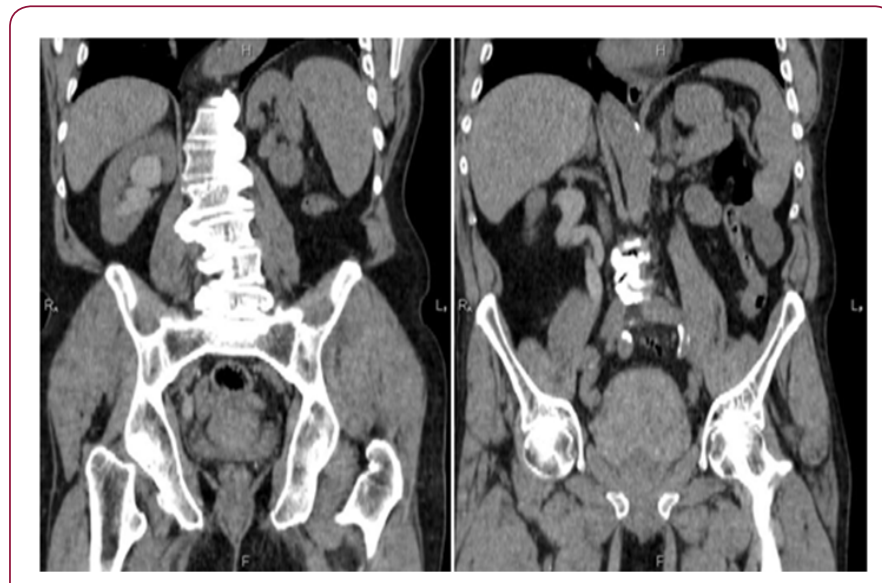

Figure 2(A-B): Reconstructed coronal images of the abdomen and pelvis showing right spontaneous renal hemorrhage reaching the urinary bladder with ipsilateral moderate hydronephrosis and hydroureter.

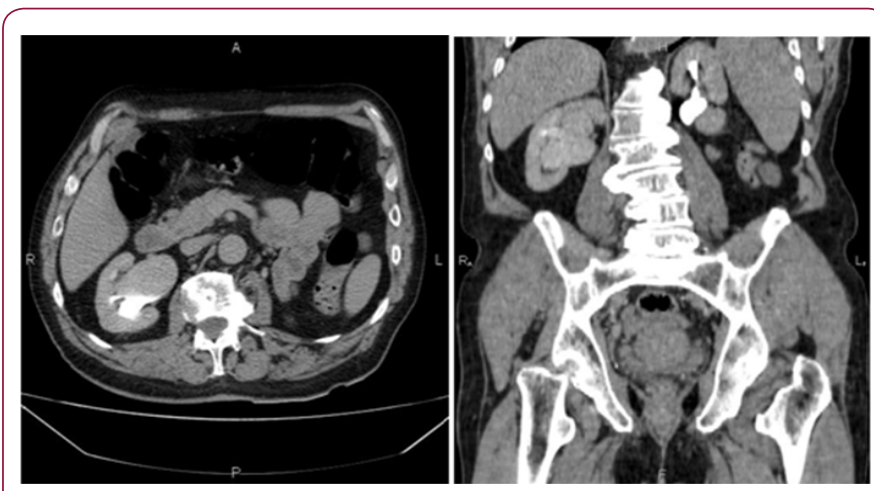

Figure 3(A-B): Axial and coronal abdominal CT images (excretory phase). A. Delayed contrast material excretion was seen in the right kidney. B. Normal excretion was seen in the left kidney.

\section{Conclusion}

Spontaneous kidney bleed can be caused by different entities like the use of antiplatelet and anticoagulation. Kidney tumors are not the offender all the time. So nephrectomy should not be done for all patients since conservative management sometimes will control the bleed and save the kidneys.

\section{References}

1. Pode D, Meretik S, Shapiro A (1985) Diagnosis and management of renal angiomyolipoma. Urology 25: 461-467.

2. Srinivasan V, Turner AG, Blackford HN (1994) Massive intraperitoneal hemorrhage associated with renal pathology. J Urol 151(4): 980-981.

3. Hayman J, Leiter E (1987) Spontaneous retroperitoneal hemorrhage: unusual presentation of renal cell cancer. Urology 30: 259-261.

4. Spires AM, Gaede JT, Glenn JF (1980) Death from renal cyst. Urology 16: 606-607.

5. Buller HR, Agnelli G, Hull RD (2004) Antithrombotic therapy for venous thromboembolic disease: the Seventh ACCP Conference on Antithrombotic and Thrombolytic Therapy. Chest 126(3): 401S-428S.

6. Topgul K, Uzun O, Anadol AZ, Gok A (2005) Surgical management of enoxaparin-and /or warfarin-induced massive retroperitoneal bleeding: report of a case and review of the literature. South Med J 98(1): 104-106. 
7. Mrug M, Mishra PV, Lusane HC, Cunningham JM, Alpert MA (2002) Hemothorax and retroperitoneal hematoma after anticoagulation with enoxaparin. South Med J 95(8): 936-938.

8. Chan-Tak KM (2003) Fatal retroperitoneal hematoma secondary to enoxaparin. South Med J 96: 58-60.

9. Daliakopoulos SI, Bairaktaris A, Papadimitriou D, Pappas P (2008) Gigantic retroperitoneal hematoma as a complication of anticoagulation therapy with heparin in therapeutic doses: a case report. J Mexd Case Reports 2: 162 .
10. Belville JS, Morgentaler A, Loughlin KR, Tumeh SS (1989) Spontaneous perinephric and subcapsular renal hemorrhage: Evaluation with CT, US, and angiography. Radiology 172(3): 733-738.

11.Sherer DM, Dayal AK, Schwartz BM, Oren R, Abufalia O (1999) Extensive spontaneous retroperitoneal hemorrhage: an unusual complication of heparin anticoagulation during pregnancy. J Matern Fetal Med 8: 196199

12.Amano T, Takemae K, Niikura S, Kouno M, Amano M (1999) Retroperitoneal hemorrhage due to spontaneous rupture of adrenal myelolipoma. Int J Urol 6(11): 585-588.

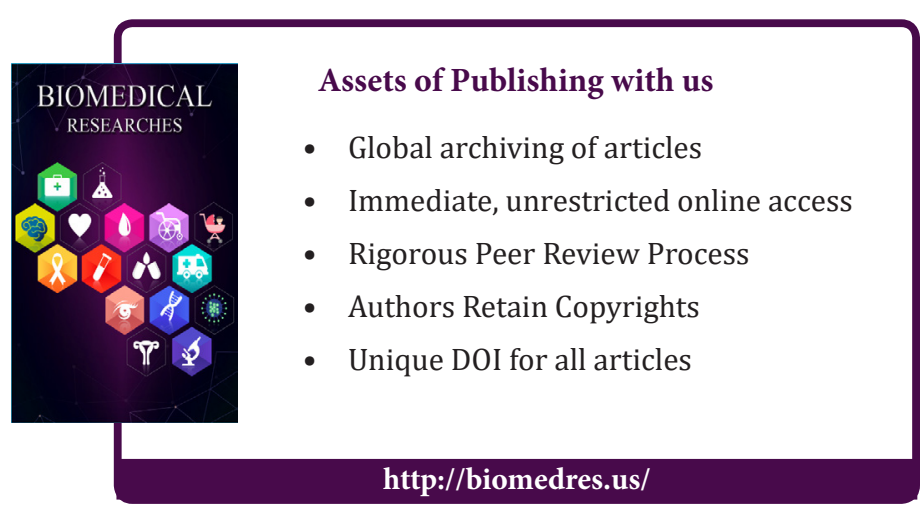

